Supporting information for

\title{
Multidimensional Digital Bioassay Platform Based on an Air-Sealed Femtoliter Reactor Array Device
}

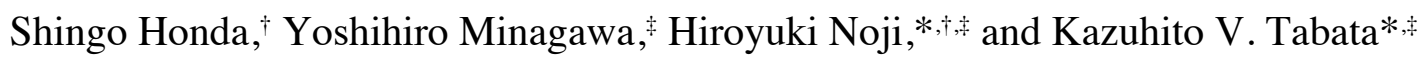

${ }^{\dagger}$ Department of Bioengineering, ${ }^{\star D}$ epartment of Applied Chemistry, Graduate School of

Engineering, The University of Tokyo, 7-3-1 Hongo, Bunkyo-ku, Tokyo 113-8656, Japan

*Corresponding authors: Hiroyuki Noji and Kazuhito V. Tabata

Email: hnoji@g.ecc.u-tokyo.ac.jp

ktabata@smb.t.u-tokyo.ac.jp

This PDF file includes:

Figures S1 to S9

Supplementary methods

Supplementary references 

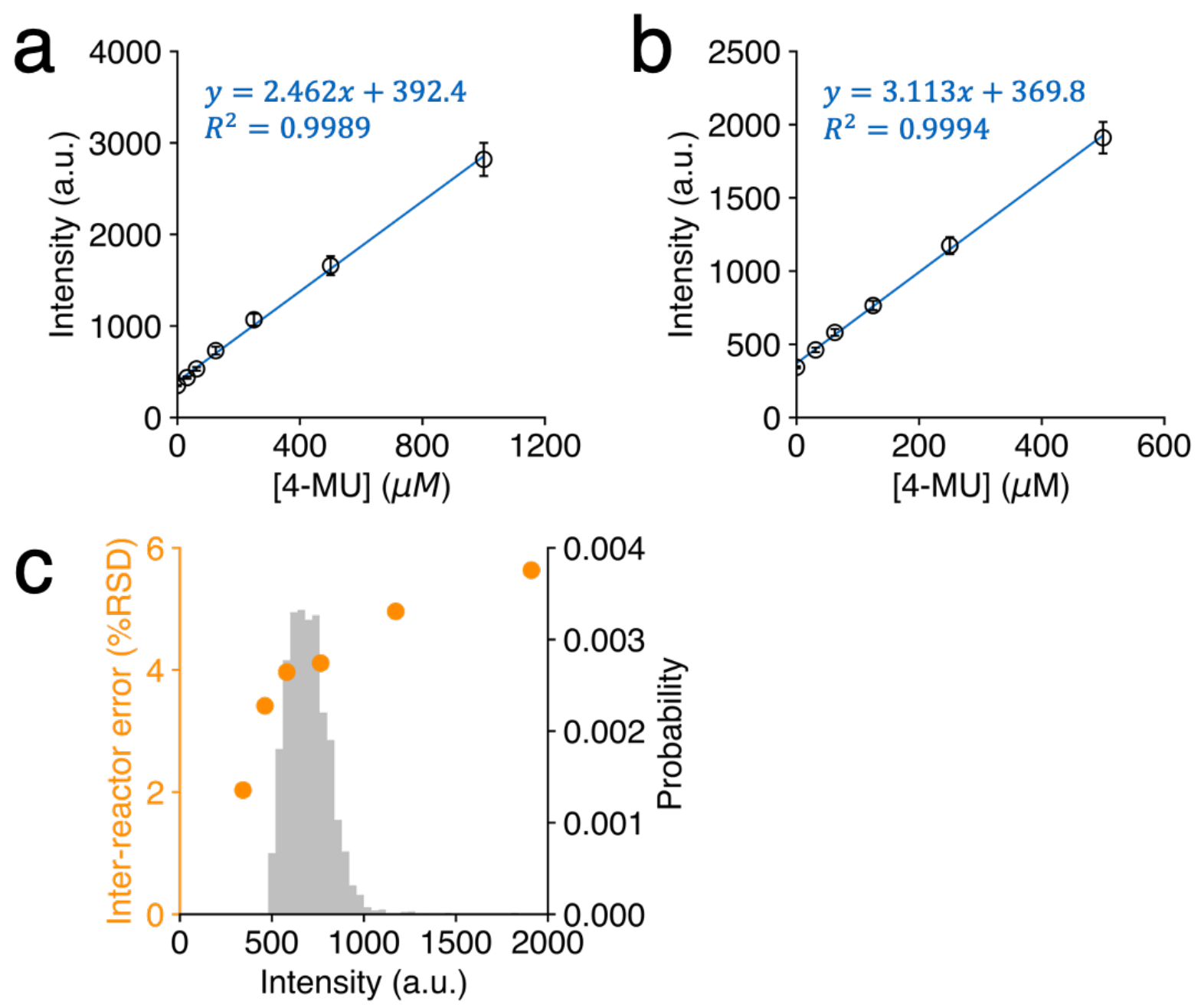

Figure S1. Calibration curve of 4-MU in assay buffer A (a) and assay buffer B (b). The mean \pm standard deviation (SD) of the fluorescence intensity in the reactors is plotted against the dose of 4-MU. (a) $n>190000$ and (b) $n>220000$. (c) Inter-reactor error (corresponding to \% RSD of the fluorescence intensity in (b)) is plotted against the fluorescence intensity of 4-MU in a reactor (orange). The gray histogram shows the distribution of fluorescence intensity of 4-MU in reactors at $t=4 \mathrm{~min}$ in the NA turnover measurement. 


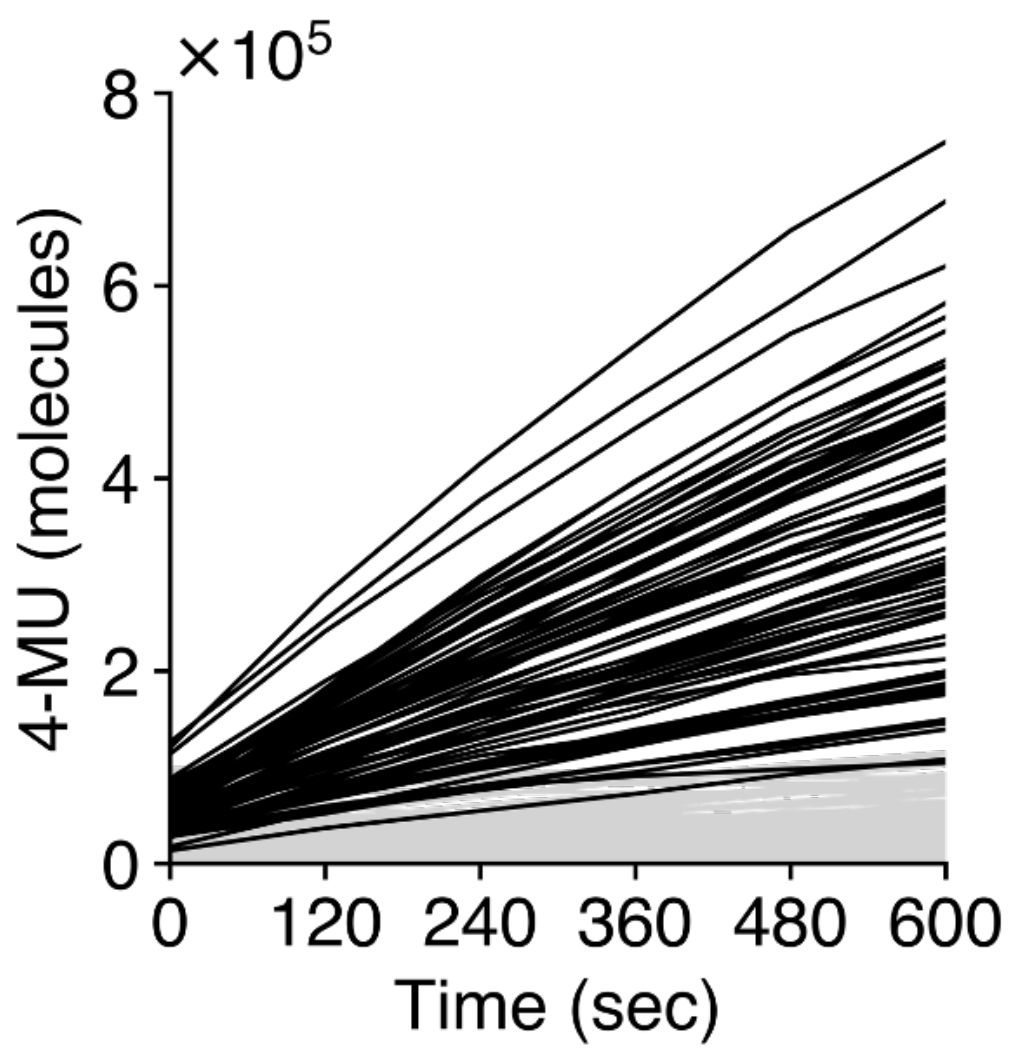

Figure S2. Change in the number of 4-MU molecules in typical reactors over time. Black and gray plots represent IAV particles of which NA turnovers are above and below the threshold, respectively, as defined in Figure 3c. 


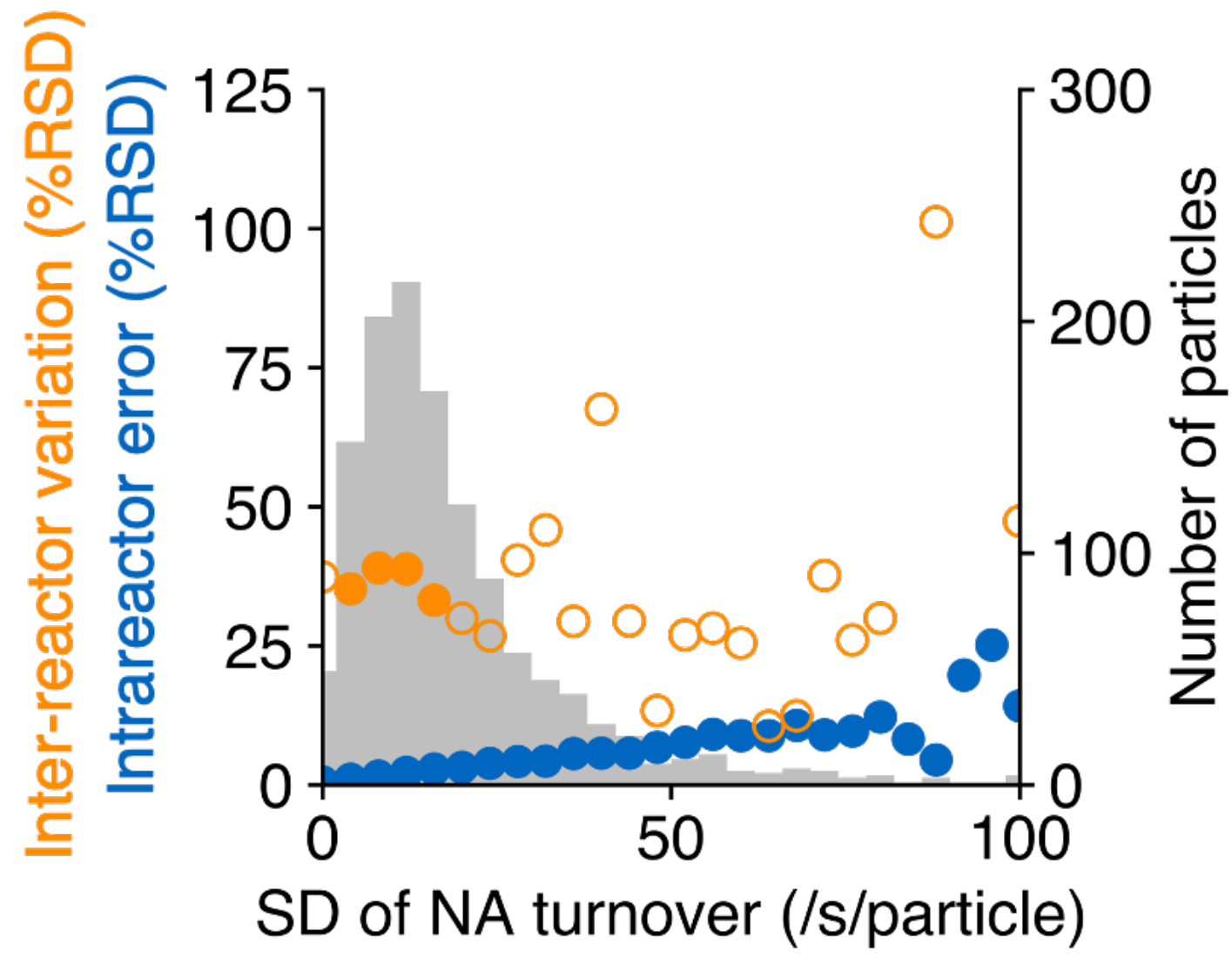

Figure S3. Inter-reactor variation and intrareactor error in NA turnover measurement estimated by one-way ANOVA. Gray: number of virus particles in each subpopulation sliced with the SD of NA turnover measurement. Blue: intrareactor error of each subpopulation. Orange: interreactor variation in each subpopulation (Subpopulations that have more than $10 \%$ of the original population are shown in closed circles). 


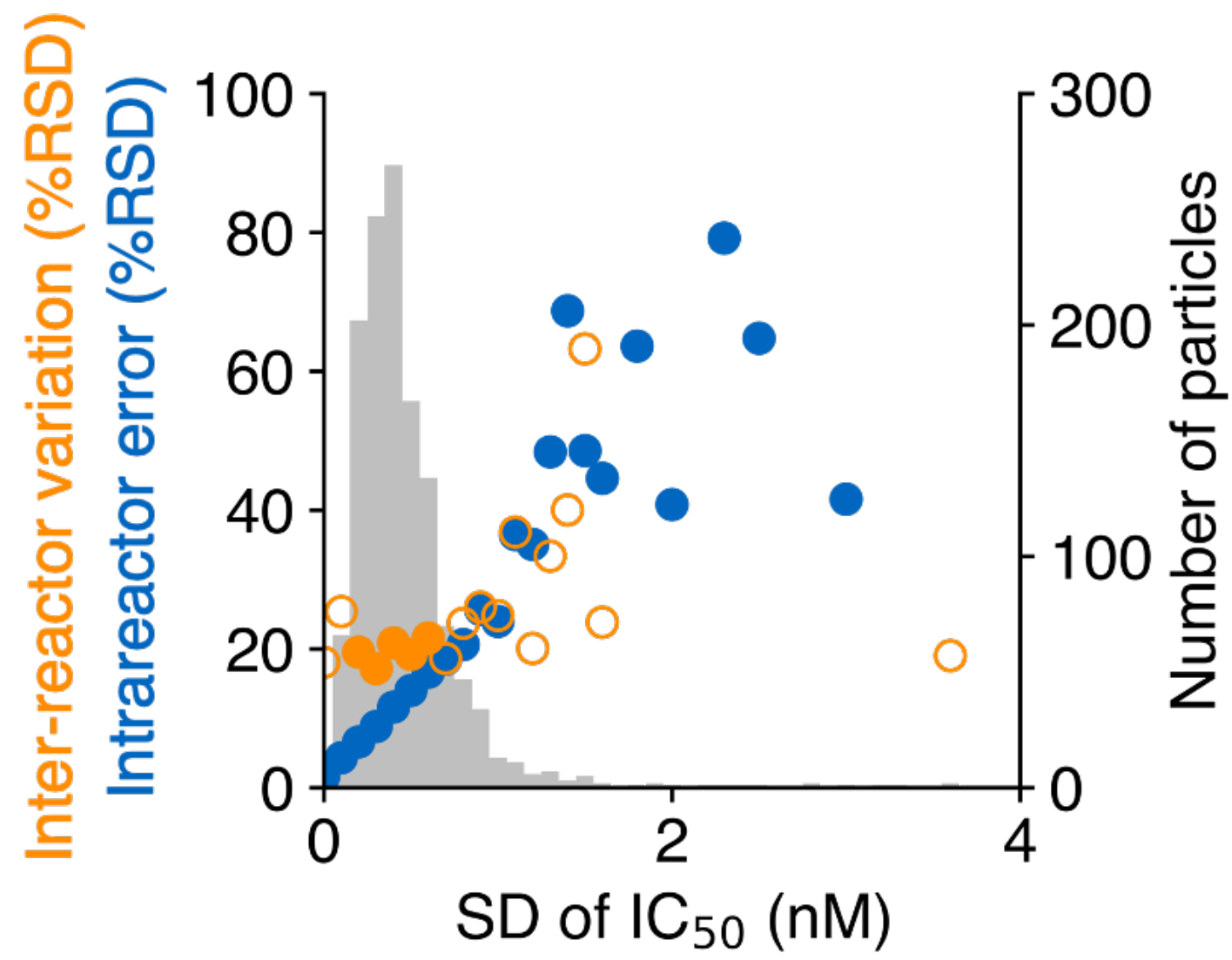

Figure S4. Inter-reactor variation and intrareactor error in $\mathrm{IC}_{50}$ measurement of oseltamivir estimated by one-way ANOVA. Gray: number of virus particles in each subpopulation sliced with the $\mathrm{SD}$ of the $\mathrm{IC}_{50}$ measurement. Blue: intrareactor error in each subpopulation. Orange: inter-reactor variation in each subpopulation (Subpopulations that have more than $10 \%$ of the original population are shown in closed circles). 

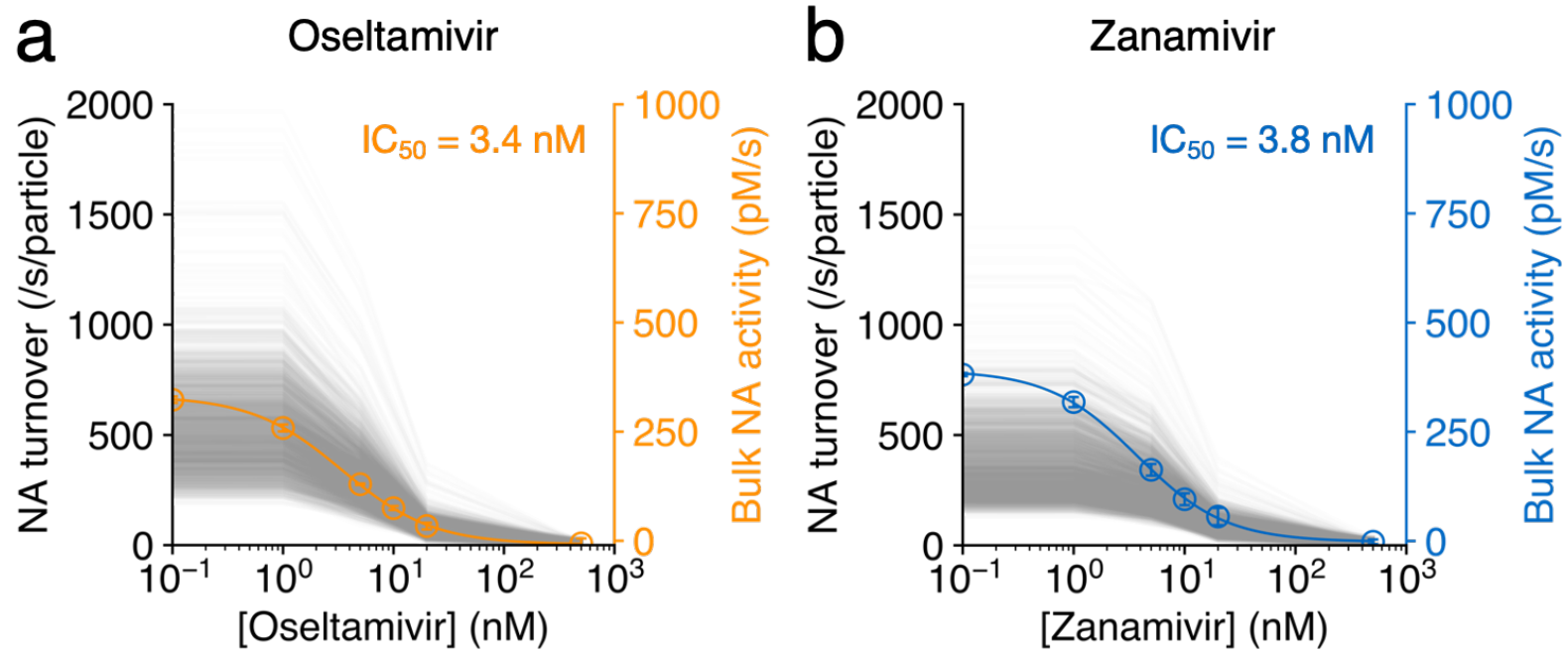

Figure S5. NAI dose-dependency of NA turnover. (a) Oseltamivir or (b) zanamivir dosedependent decrease in NA turnover is shown for 2286 virus particles. Each gray plot represents a single virus particle. The mean \pm SD of NA turnover measured in a conventional bulk scale bioassay are plotted versus (a) oseltamivir or (b) zanamivir concentration and fitted with the Hill equation to calculate the $\mathrm{IC}_{50}$. The resulting $\mathrm{IC}_{50}$ is shown in each figure. 

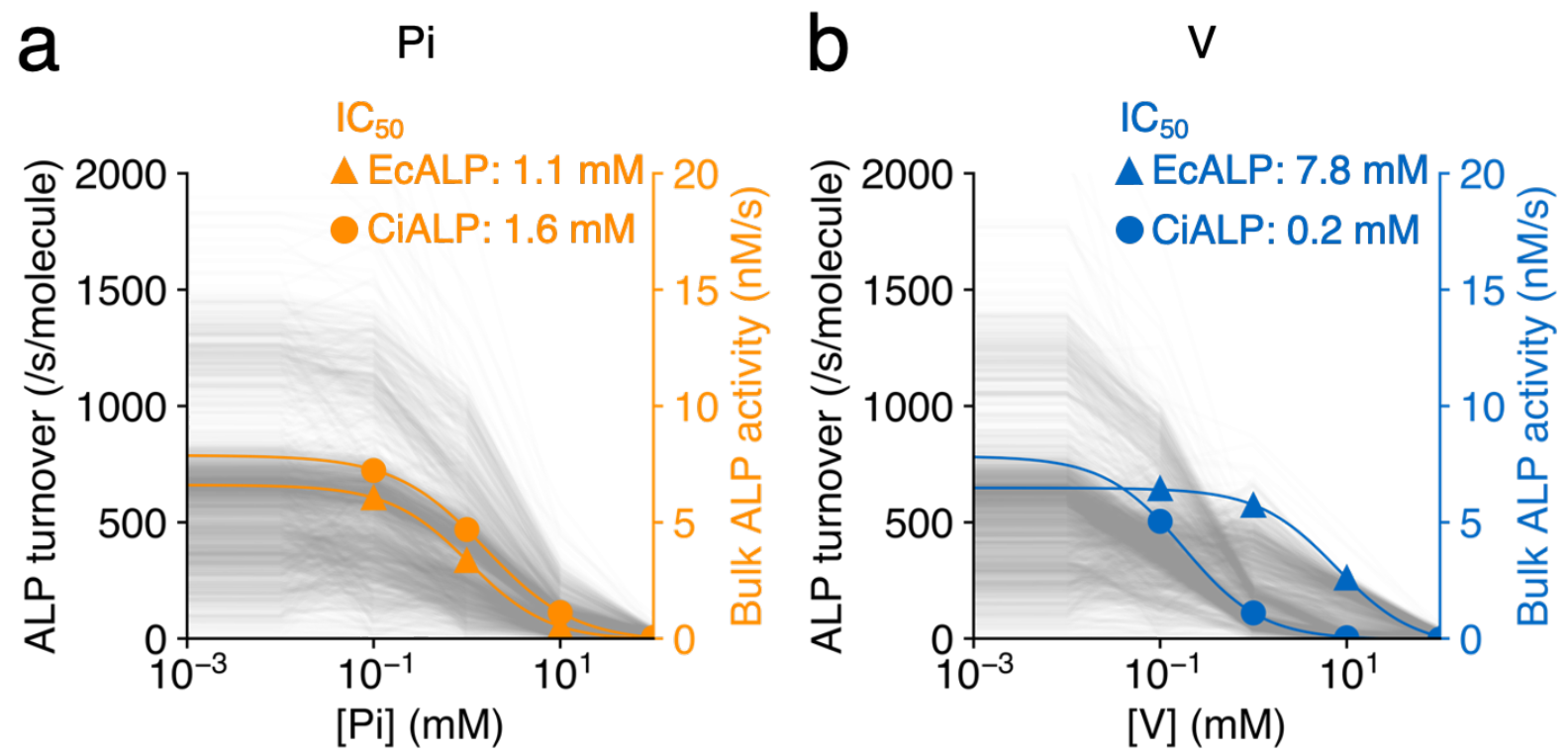

Figure S6. Inhibitor dose-dependency of ALP turnover. (a) Pi or (b) V dose-dependent decrease in ALP turnover is shown for 2200 enzyme molecules of ALP mix. Each gray plot represents a single enzyme molecule. The mean \pm SD of ALP turnover measured in a conventional bulk scale bioassay of EcALP or CiALP are plotted versus (a) Pi or (b) V concentration and fitted with the Hill equation to calculate the $\mathrm{IC}_{50}$. The resulting $\mathrm{IC}_{50}$ is shown in each figure. 


\section{a}
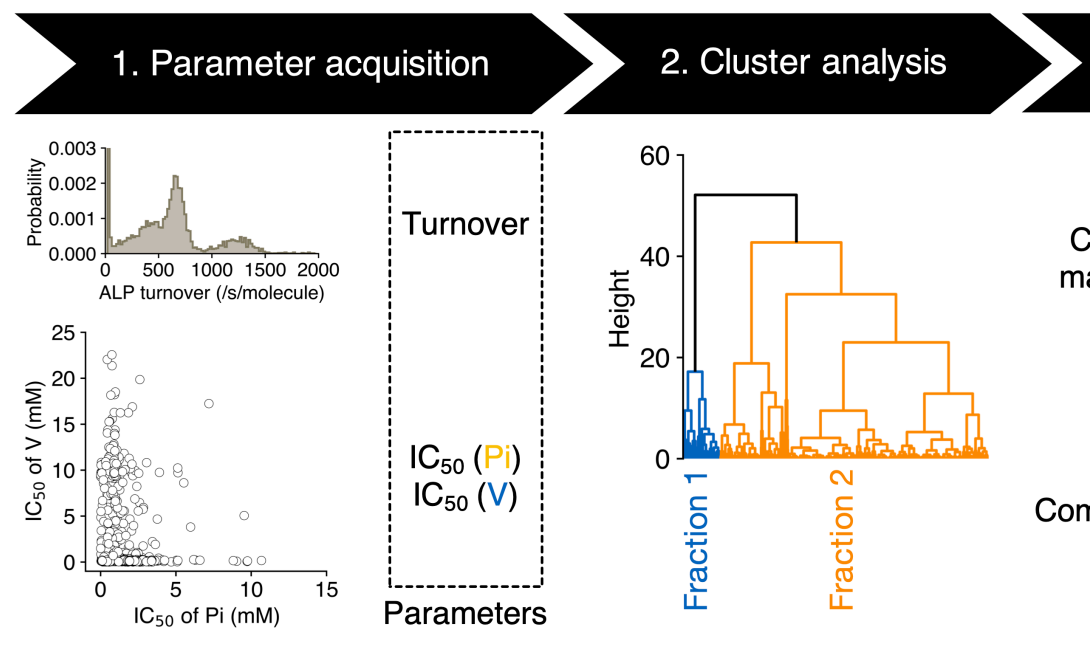

3. Comparison with result

from conventional bioassay

\section{b}
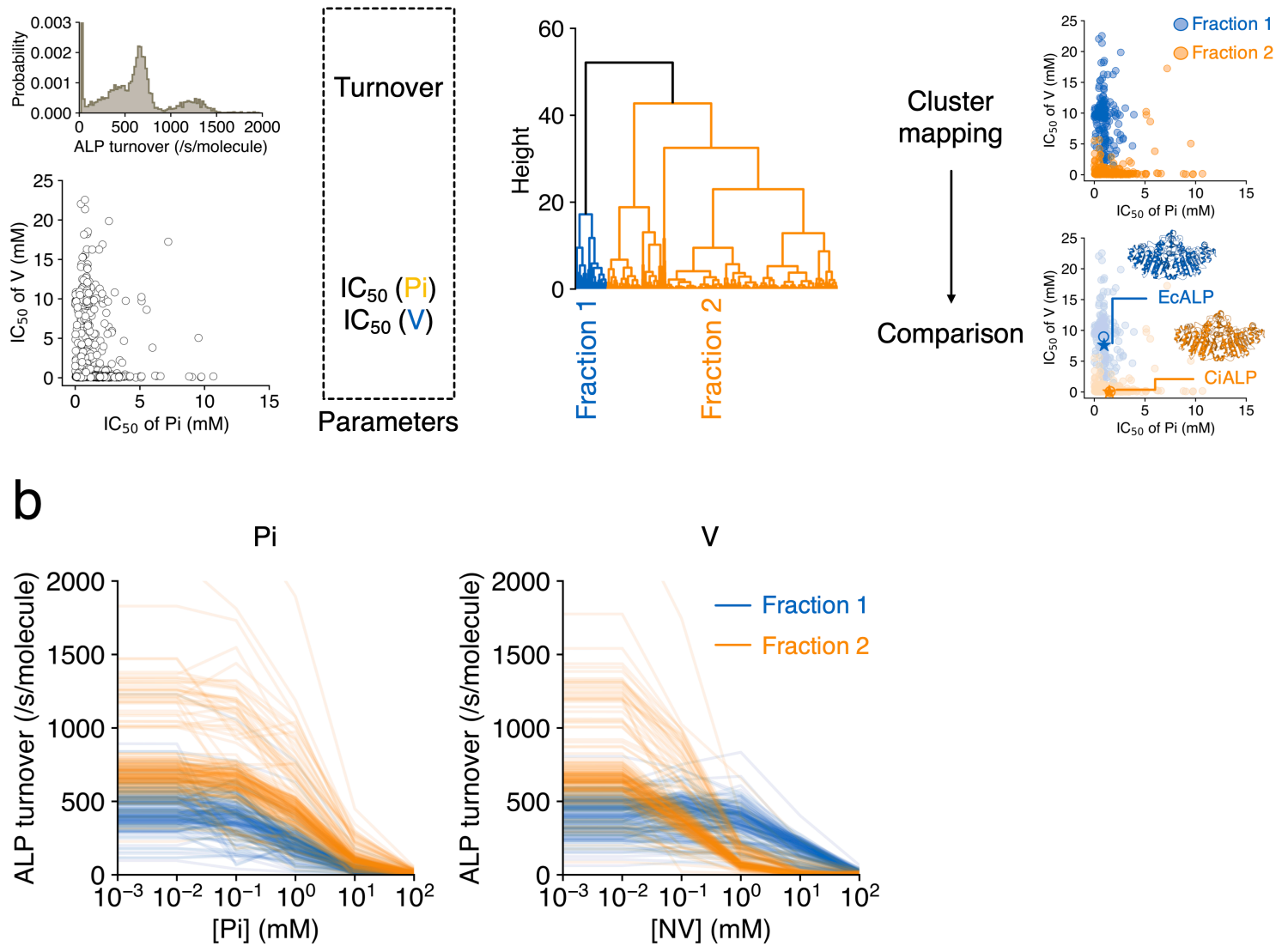

Figure S7. Discrimination of ALPs of two origins with multiparametric assay and cluster analysis. (a) Schematic of the experiment and analysis. (1) Three parameters for cluster analysis (i.e., turnover and $\mathrm{IC}_{50} \mathrm{~s}$ ) were acquired by a turnover measurement (top) and $\mathrm{IC}_{50}$ measurements (bottom) of ALP mix. (2) Cluster analysis was performed and two fractions were defined from the resulting dendrogram. (3) The fractions were mapped on the plot of $\mathrm{IC}_{50} \mathrm{~s}$ (top). Then the centers of the two fractions were calculated (bottom, shown as blue and orange open circles). The centers were compared with the $\mathrm{IC}_{50} \mathrm{~s}$ measured in conventional bulk scale bioassays (bottom, shown as blue and orange stars). (b) Two fractions of ALP molecules shown as inhibition curves. 1444 enzyme molecules resulting from the cluster analysis are plotted. 

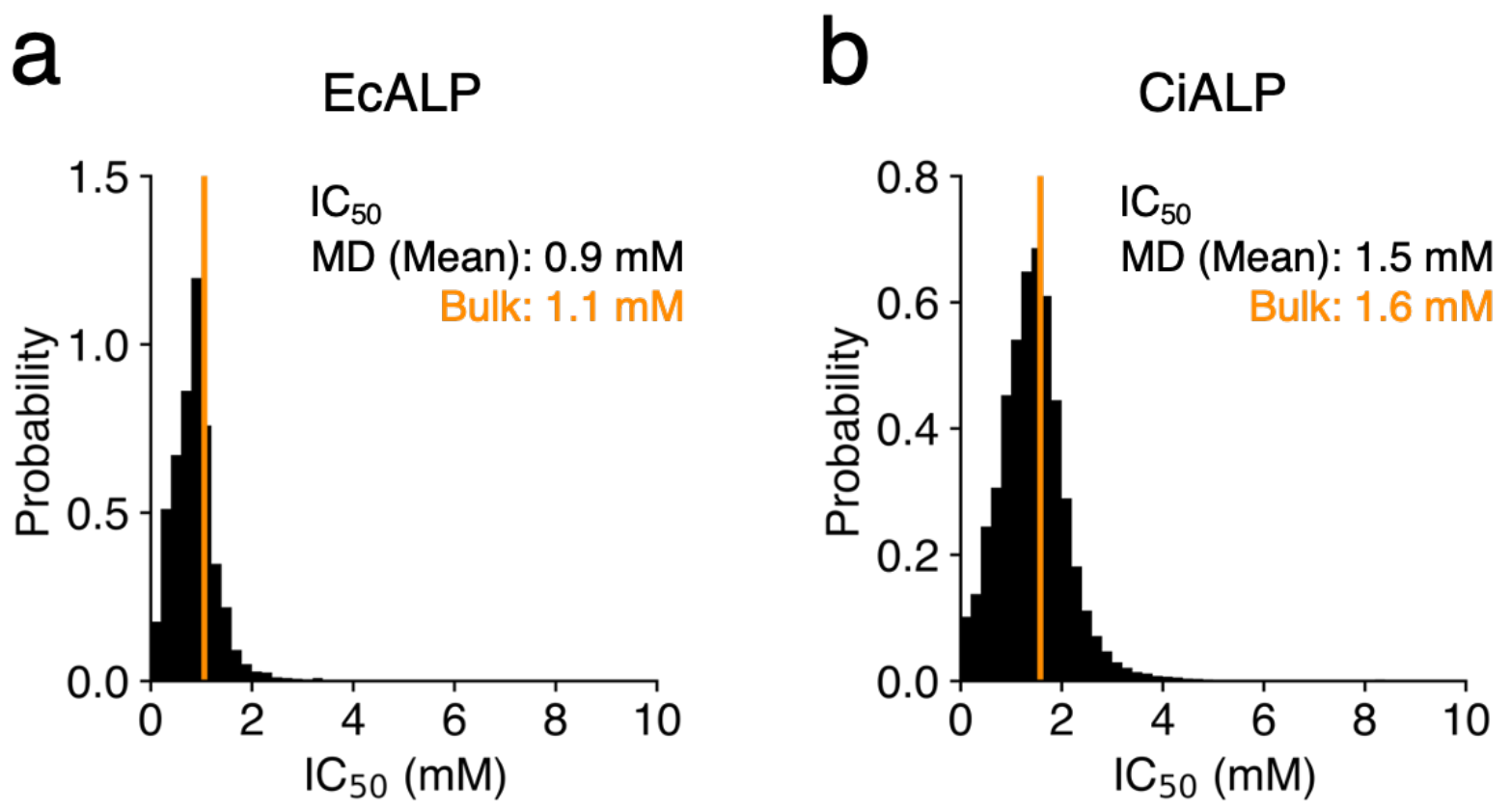

Figure S8. $\mathrm{IC}_{50} \mathrm{~S}$ of $\mathrm{Pi}$ for $\mathrm{ALP}$ measured in MD digital bioassays and conventional bulk scale bioassays. Distribution of $\mathrm{IC}_{50}$ of Pi for (a) EcALP molecules $(n=500)$ and (b) CiALP molecules $(n=1107)$. Orange line: $\mathrm{IC}_{50}$ s measured in conventional bulk scale bioassays. 
a

Air-sealed

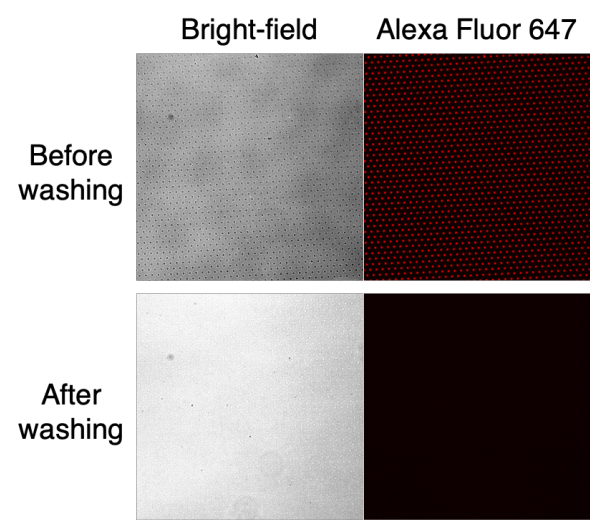

Oil-sealed

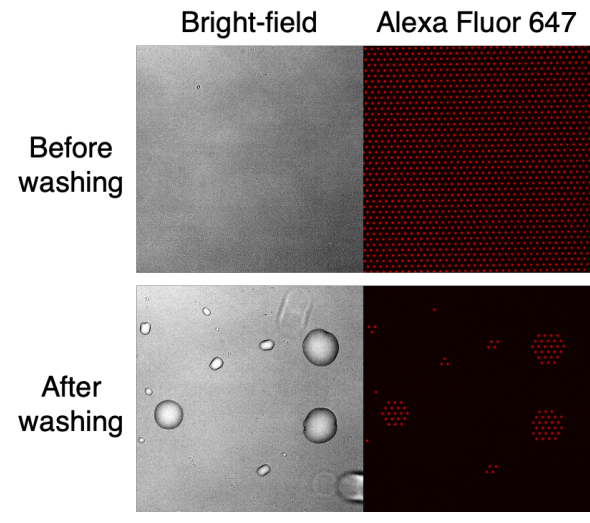

b

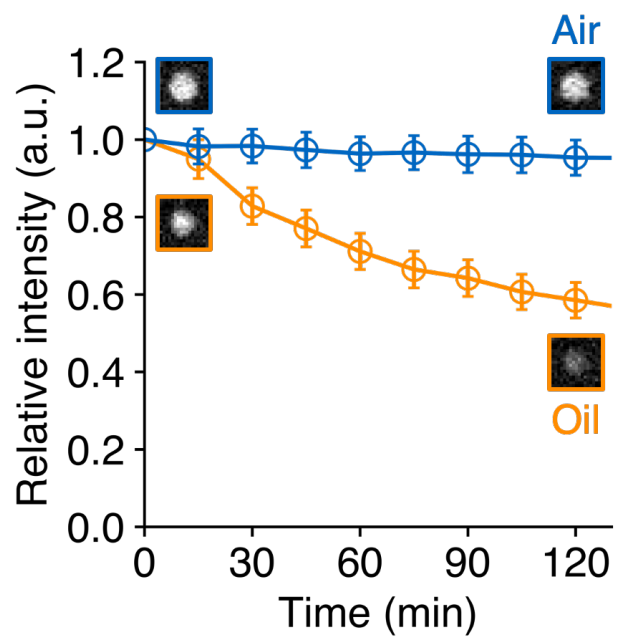

Figure S9. Comparison of air-sealing and conventional oil-sealing. (a) Integrity of the reactors after washing. $10 \mu \mathrm{M}$ Alexa Fluor 647 dye in assay buffer A containing 0.02\% (v/v) TWEEN20 was loaded into the reactors and sealed with air (top) or mineral oil (bottom). After acquiring a bright-field image and a fluorescent image of Alexa Fluor 647 (Before washing), the flow channel was washed sequentially with $20,80,200$, and $200 \mu \mathrm{L}$ buffer. Then images were acquired again with the buffer filled in the flow channel (After washing). (b) Leakage of hydrophobic fluorescent dye 4-MU from reactors over time. $50 \mu \mathrm{M}$ 4-MU was loaded into $3.4 \mathrm{fL}$ reactors and sealed $\left(1.0 \times 10^{5}\right.$ molecules/reactor $)$. Air: sealed with air. Oil: sealed with fluorocarbon oil FC-40. The fluorescent intensity relative to that at $t=0 \mathrm{~min}$ is plotted after background subtraction and correction of photobleaching. 


\section{SUPPLEMENTARY METHODS}

\section{Data analysis}

(1) Calculation of Fluorescence Intensity of a Dye in a Reactor. We calculated the fluorescence intensity of 4-MU and Alexa Fluor 647 in each reactor recorded in each experiment using a custom ImageJ macro. Briefly, we applied a Gaussian filter to the fluorescent images of Alexa Fluor 647 and defined the top of each peak corresponding to each reactor as the center of the reactor. We drew a 10 pixels x 10 pixels square around this center to surround the reactor. The fluorescence intensity of 4-MU or Alexa Fluor 647 averaged over the surrounded area was defined as the mean fluorescence intensity of the dye in the reactor. Note that, in the validation of solution stability, the fluorescence intensity at the central pixel was defined as the fluorescence intensity of the dye in the reactor.

(2) Validation of Solution Stability, Independence, and Exchangeability. In the validation of solution stability (Figure 2a), the fluorescence intensity of Alexa Fluor 647 in each reactor at each time point was normalized to that at $t=0 \mathrm{~min}$. The mean \pm standard deviation (SD) of the normalized fluorescence intensity of Alexa Fluor 647 in the reactors was plotted versus time.

In the validation of solution independence (Figure $2 b$ ), the mean $\pm \mathrm{SD}$ of the fluorescence intensity of Alexa Fluor 647 of the reactors was plotted versus time before and after photobleaching.

In the validation of solution exchangeability (Figure 2c), the distribution of the fluorescence intensity of 4-MU in the reactors was fitted by a Gaussian function to calculate the mean \pm SD. The mean \pm SD was plotted versus the rounds of solution exchanges. 
(3) Enzyme Activity Analysis in FRAD. In the NA and ALP turnover measurements, we excluded improperly sealed reactors, typically fused to large droplets formed outside the reactors, from the subsequent analyses as follows: The distribution of the fluorescence intensity of Alexa Fluor 647 in the reactors was fitted with a Gaussian function to calculate the mean $\pm \mathrm{SD}$. Since improperly sealed reactors show higher fluorescence intensity than properly sealed ones, we set a threshold (mean $+3 \mathrm{SD}$ ) and excluded the reactors above this.

We converted the fluorescence intensity of 4-MU to the number of 4-MU molecules in a reactor using the calibration curve of 4-MU (Figure S1a,b) and the reactor volume (3.4 fL). The numbers of 4-MU molecules at $t=0,2$, and 4 min were fitted with the least-square method to calculate the slope, that is, the turnover of a single entity (IAV particle or ALP molecule). We plotted histograms for the distributions of NA and ALP turnover and defined the peak around 0 as background, which corresponds to the reactors without entities. We calculated the mean \pm SD of the background peak by Gaussian fitting. We set a threshold (NA, mean +15 SD; ALP, mean $+3 \mathrm{SD}$ ) in all the turnover measurements without an inhibitor and excluded the reactors below it as background, that is, without entities. For the subsequent analyses, we used the reactors fulfilling the threshold in all the measurements without an inhibitor (1304 reactors in Figure 3, 4, Figure S3, S4; 2286 reactors in Figure 5b, Figure S5; 2200 reactors in Figure 6b, Figure S6; 1672 reactors in Figure S8a; 2098 reactors in Figure S8b). In each reactor and at each dose of NAI, mean, SD, and RSD of NA turnover were calculated from triplicate measurements.

(4) Measurement Error Estimation of NA Turnover. We estimated measurement error and intrinsic variation of NA turnover among 1304 IAV particles with one-way ANOVA (Figure 3d, Figure S3). To prepare sufficient datasets for ANOVA, we employed bootstrapping. We resampled the NA turnover measured with $i(\mathrm{nM})$ oseltamivir $(i=0,1,5,20,500)$ in the $j$ th 
reactor $(j=1,2,3, \ldots, 1304) 100$ times for each $i$ and $j$. The detailed procedure is as follows: Based on the mean \pm SD of NA turnover determined for a set of $i$ and $j$ from the triplicate measurements, we generated a random number following a normal distribution $N\left(\right.$ mean, $\left.\mathrm{SD}^{2}\right)$. We repeated this 100 times and named the $k$ th random number generated as " $v_{i, j, k}$ " $(k=1,2,3, \ldots$, 100). $v_{i, j, k}(k=1,2,3, \ldots, 100)$ was employed as a virtual NA turnover dataset for each $i$ and $j$.

We used $v_{0, j, k}$ for the measurement error estimation of NA turnover. The variance of $v_{0, j, k}$ consists of intrareactor error (among $v_{0, j, 1}, v_{0, j, 2}, v_{0, j, 3}, \ldots v_{0, j, 100}$ ) and inter-reactor variation (among $v_{0,1, k}, v_{0,2, k}, v_{0,3, k}, \ldots, v_{0,1304, k}$ ). The intrareactor error is equivalent to measurement repeatability, and the inter-reactor variation is derived from intrinsic variation and inter-reactor error among 1304 virus particles. The inter-reactor error is attributed to error from the imaging system and variation in reactor volume.

Although we first tried to separate the inter-reactor variation from the intrareactor error with one-way ANOVA, we could not because the measurement repeatability differed among the reactors (Figure 3d), which meant the intrareactor error (SD) of NA turnover in each reactor was not homogeneous. Therefore, we "sliced" 1304 virus particles into subpopulations by the SD of NA turnover in each reactor at an SD increment of 4 turnovers/s/particle. Then, one-way ANOVA was applied to each subpopulation (Figure S3), assuming that the SD of NA turnover in the subpopulation in each reactor was homogeneous. We estimated the inter-reactor variation (orange circles in Figure S3) separate from the intrareactor error (blue circles in Figure S3). We regarded the estimated inter-reactor variation in subpopulations with more than $10 \%$ of the original virus particle population ( $\geq 131$, closed orange circles in Figure S3) as sufficiently reliable (33\% to 39\% RSD). The inter-reactor error depends on the fluorescence intensity of 4MU in each reactor (Figure S1c). As mentioned above, NA turnover was determined by fitting 
fluorescence intensity of 4-MU in a reactor at $t=0$ to $4 \mathrm{~min}$. Given that the fluorescence intensity at $t=4 \min (700 \pm 130)$ was the highest and therefore mostly controlled the fitting, the inter-reactor error can be estimated to be 5\% RSD at most (Figure S1c). Even after subtraction of this inter-reactor error from the inter-reactor variation, the intrinsic variation among the virus particles was 33\% to 39\% RSD, which suggested the inter-reactor error had little impact on the result.

(5) NAI Sensitivity Analysis in FRAD and the Measurement Error Estimation. We determined $\mathrm{IC}_{50} \mathrm{~S}$ of NAI on individual IAV particles and estimated the measurement error and the intrinsic variation among 1304 IAV particles with one-way ANOVA (Figure 4b inset, 4c, Figure S4). For this purpose, we used the virtual NA turnover dataset $v_{i, j, k}$, where $i, j$, and $k$ stand for the dose of oseltamivir $(i=0,1,5,20,500)$, reactor number $(j=1,2,3, \ldots, 1304)$, and resampling number $(k$ $=1,2,3, \ldots, 100)$, respectively. We fitted $\left(\left[v_{0, j, k}\right],\left[v_{1, j, k}\right],\left[v_{5, j, k}\right],\left[v_{20, j, k}\right],\left[v_{500, j, k}\right]\right)$ with the Hill equation and obtained an $\mathrm{IC}_{50}$ value for each $j$ and $k$, which we named $\mathrm{IC}_{50 j, k}$. After excluding four reactors that we failed to fit, we calculated the mean (Figure 4b inset), SD, and RSD (Figure 4c) of the $\mathrm{IC}_{50}$ values $\left(\mathrm{IC}_{50 j, 1}, \mathrm{IC}_{50 j, 2}, \mathrm{IC}_{50 j, 3}, \ldots, \mathrm{IC}_{50 j, 100}\right)$ for each $j$. The variance of $\mathrm{IC}_{50 j, k}$ consists of intrareactor error (among $\mathrm{IC}_{50 j, 1}, \mathrm{IC}_{50 j, 2}, \mathrm{IC}_{50 j, 3}, \ldots \mathrm{IC}_{50 j, 100}$ ) and inter-reactor variation (among $\mathrm{IC}_{501, k}, \mathrm{IC}_{502, k}, \mathrm{IC}_{503, k}, \ldots, \mathrm{IC}_{501300, k}$ ). The intrareactor error is equivalent to measurement repeatability, including fitting error, and the inter-reactor variation directly reflects the intrinsic variation of $\mathrm{IC}_{50}$ among the 1300 virus particles, as the inter-reactor error is mostly canceled out in the process of fitting. As measurement repeatability differed among the reactors (Figure 4c), we "sliced" the 1300 virus particles into subpopulations by the $\mathrm{SD}$ of $\mathrm{IC}_{50}$ in each reactor at an SD increment of $0.1 \mathrm{nM}$, as we did with NA turnover in the previous experiment. Then, one-way ANOVA was applied to each subpopulation (Figure S4), assuming that the SD of $\mathrm{IC}_{50}$ in the 
subpopulation in each reactor was homogeneous. We estimated the intrinsic variation (orange circles in Figure S4) separate from the intrareactor error (blue circles in Figure S4). We regarded the estimated intrinsic variation in subpopulations with more than $10 \%$ of the original virus particle population ( $\geq 131$, closed orange circles in Figure S4) as sufficiently reliable (17\% to $22 \% \mathrm{RSD})$. The intrinsic variation of $\mathrm{IC}_{50}$ in all these subpopulations were significant and could not be explained by the measurement error ( $\mathrm{P}<0.001$, one-way ANOVA).

(6) ALP Inhibitor Sensitivity Analysis in FRAD. We determined $\mathrm{IC}_{50} \mathrm{~s}$ of ALP inhibitors (Pi and V) for individual ALP molecules by fitting the ALP turnovers measured at each dose of an inhibitor $(0,0.01,0.1,1,10$, and $100 \mathrm{mM})$ in each reactor (2200 reactors) with the Hill equation. Variation of calculated $\mathrm{IC}_{50} \mathrm{~s}$ for individual ALP molecules was higher than that calculated for individual IAV particles. Moreover, we failed to fit a greater number of reactors than we did in the case of IAV particles. This was likely due to the stochastic feature of a single-molecule experiment. A single-molecule ALP turnover measurement is usually accompanied by the high variability caused by stochastic fluctuation in the enzyme structure, while in the NA turnover measurements on a single IAV particle, an IAV particle has approximately 50 NA tetramers; NA turnover was averaged over the particle, which reduced the variation. To mitigate the impact of the $\mathrm{IC}_{50}$ variability on the subsequent cluster analysis, we set a threshold (the coefficient of determination for the fitting, $\mathrm{R}^{2}>0.9$ and $\mathrm{IC}_{50}<25 \mathrm{mM}$ ) and excluded the reactors that did not meet this threshold. Note that the $\mathrm{IC}_{50}$ variability was considered to be mainly due to the fitting error caused by the random variation in ALP turnover measurements, which means it is unlikely that the thresholding caused certain biases in the ALP population.

(7) Enzyme Activity Analysis and Inhibitor Sensitivity Analysis on a Microtiter Plate. We calculated the 4-MU concentrations of the samples at each time point from the 4-MU 
fluorescence intensity and the calibration curves (Figure S1a,b). Then, we fitted the 4-MU concentrations at 1 to $5 \mathrm{~h}$ (NA) or at 0 to $15 \mathrm{~min}$ (ALP) with the least-square method to determine the enzyme activity. The enzyme activity at each dose of inhibitor was fitted with the Hill equation to determine $\mathrm{IC}_{50}$ (Figure 4b, Figure S5, S6).

(8) Cluster Analysis of ALP Mix. We normalized the ALP turnover determined without an inhibitor and the $\mathrm{IC}_{50} \mathrm{~s}$ of $\mathrm{Pi}$ and $\mathrm{V}$ in each reactor and used them as input parameters for cluster analysis. The cluster analysis was performed using a clustering package scipy.cluster.hierarchy implemented in Python with SciPy;

https://docs.scipy.org/doc/scipy/reference/cluster.hierarchy.html. With the hierarchical Ward's method $^{1}$ based on the Euclidean distance, we separated the population of ALP mix into two fractions (Figure 6a bottom, 6b, Figure S7). 


\section{SUPPLEMENTARY REFERENCES}

(1) Ward, J. H. J. Am. Stat. Assoc. 1963, 58 (301), 236-244. 